\title{
IoT Design Space Challenges: Circuits and Systems
}

\author{
D. Blaauw, D. Sylvester, P. Dutta, Y. Lee, I. Lee, S. Bang, Y. Kim, G. Kim, P. Pannuto, \\ Y.-S. Kuo, D. Yoon, W. Jung, Z. Foo, Y.-P. Chen, S. Oh, S. Jeong, M. Choi \\ University of Michigan, Ann Arbor, MI, USA
}

\begin{abstract}
The Internet of Things (IoT) is a rapidly emerging application space, poised to become the largest electronics market for the semiconductor industry. IoT devices are focused on sensing and actuating of our physical environment and have a nearly unlimited breadth of uses. In this paper, we explore the IoT application space and then identify two common challenges that exist across this space: ultra-low power operation and system design using modular, composable components. We survey recent low power techniques and discuss a low power bus that enables modular design. Finally, we conclude with three example ultra-low power, millimeter-scale IoT systems.
\end{abstract}

\section{IoT Application Space and Challenges}

IoT devices are unique in that they are focused on physical interfaces, allowing them to sense and actuate the world around us. This is in contrast to previous computing platforms, such as desktop and handheld devices, which have primarily focused on human interfaces. These human interfaces require relatively large input and output devices and require the devices to be co-located with their users. In contrast, IoT devices receive input through sensors that are often highly miniaturized through MEMS technology and send out information through wired or wireless interfaces to mobile and cloud computers. This enables the placement of IoT devices in myrad new locations and applications where computing was previously absent. As a result, IoT presents the semiconductor industry with a market opportunity that may exceed that of all previous computing classes.

The diversity of our physical environment results in a similarly diverse IoT application space that ranges from tiny implanted heart-rhythm monitors, to large, extremely long lifetime HVAC sensors, to sensors for oil reservoir diagnosis [1]. To categorize this wide application space we recognize four ontological properties that are critical in determining IoT device requirements: sensing modality, form factor (size), energy source / sensor lifetime, and connectivity. Table 1 lists example values for these properties. For instance, physical form factor can range from tiny millimeter size for implantable sensors to $10 \mathrm{~s}$ of centimeters for infrastructure monitoring. Sensing modalities can include pressure, chemical, strain, and temperature, etc. In addition, the sensing modality can be characterized by measurement frequency and possible triggers, such as a timer, a detected event in the monitored value, or an external event such as a radio message. Similarly, communication connectivity is characterized by communication interval, distance, data rate/size, etc. Clearly the listed items are not exhaustive. Each property can take a very large number of possible values resulting in a highly varied application space. Some examples include 1) tiny sensors that measure temperature every 10 minutes using a secondary battery and harvesting with infrequent communication for studying Pika's in the Rocky mountains [2]; 2) continuous monitoring of brain activity in seizure patients with a continuous RF power link and instantaneous wireless communication upon a detected event; 3) infrastructure monitoring devices with large primary batteries and many $10 \mathrm{~s}$ of years of lifetime and very infrequent communication.

Despite its diversity, the IoT application space is characterized by two overarching design concerns. First, since the location of an IoT device is constrained by the physical quantity being monitored they are frequently in locations without easy access to power. Hence, low power consumption is possibly the most universal constraint across the IoT space. Second, since it is impossible to make a customized chip for each and every application, a highly modular approach is needed where systems can be constructed by combining pre-existing chips designed to be composable, while not unduly increasing power and size. We will discuss these constraints briefly below.

\section{Low Power Circuit Solutions}

Since IoT devices are typically untethered, they must survive on a battery or on power harvested from their environment. IoT devices typically require long lifetimes, further constraining power consumption. Fig. 1 shows maximum average power as a function of battery size and required lifetime. While larger systems can afford $\mu \mathrm{W}-\mathrm{mW}$ average power, millimeter-size devices must survive on $\mathrm{nW}$ power budgets. The characteristics in Table 1 dictate what power level can be achieved and which components will be dominant. For instance, in low duty cycle systems the sensor will be mostly inactive, reducing active power consumption and making standby power the dominant component. On the other hand, systems with high bandwidth requirements will have significantly higher power budgets with the radio as the dominant factor.

We give three examples of ultra-low circuit innovations that enable IoT devices. Fig. 2 shows a low power voltage reference that is based on the difference between the threshold voltage of a high and low $\mathrm{V}_{t}$ device [3]. The key observation (equation in Fig 2) is that the temperature dependence can be cancelled by sizing of the two devices. Hence, the reference obtains a $\mathrm{TC}=19.4 \mathrm{ppm} /{ }^{\circ} \mathrm{C}$ with $\mathrm{LS}=0.033 \% / \mathrm{V}$ and PSRR $=-67 \mathrm{~dB}$ while drawing only $10 \mathrm{~s}$ of pA's.

Similar to a voltage reference, timers are a critical always-on component in most IoT devices and must be ultra-low power. Fig. 3 shows a crystal oscillator with a power consumption of $5.5 \mathrm{nW}$, marking a $\sim 5 \mathrm{X}$ improvement over previous designs [4]. The $\mathrm{XO}$ lowers the power consumption by reducing the oscillation amplitude (typically $\sim 1 \mathrm{~V}$ ) to only $\sim 150 \mathrm{mV}$. To sustain a robust oscillation, the driver stage is driven with a high $750 \mathrm{mV}$ swing signal, which is generated by amplifying the XO output node voltage. The power is further reduced by only activating the driver for a brief period when the XO input node reaches its $\mathrm{min} / \mathrm{max}$ values, thereby reducing the voltage across the driver switch and hence the power loss.

IoT devices often need to harvest energy under a range of energy conditions, requiring voltage up-conversion with highly varying input power levels. In addition, IoT device form factors make it desirable to fully integrate the DC-DC up converter on-chip. Fig. 4 shows a fully integrated voltage doubler that offers $>70 \%$ efficiency across an extremely wide current range of $1 \mathrm{nA}$ to $300 \mathrm{uA}$ [5]. By building an oscillator into the switch cap structure (using an RO topology), a dedicated clock generator and level shifters are avoided, improving efficiency. When used in a harvester, the doubler self-starts at $140 \mathrm{mV}$ input voltage and can charge a $4 \mathrm{~V}$ battery from a $1 \mathrm{~mm}^{2}$ solar cell in dim indoor lighting.

\section{Low Power Inter-die Communication and System Examples}

Due to the high diversity of IoTapplications, it is necessary to construct systems using multiple, compatible die that are interconnected through a simple communication bus. Existing standards require either a large footprint due to a high number of wires (SPI [6]) or high power due to the use of pull-up resistors $\left(\mathrm{I}^{2} \mathrm{C}\right.$ [7]). We therefore recently proposed a new bus protocol called MBus [8] that requires only two unidirectional wires, connected in a daisy chain topology (Fig. 5). The bus protocol reduces active power by eliminating the need for a clock generator on each slave layer and employs extensive power gating to reduce sleep power.

Using the discussed low power techniques and Mbus inter-die communication, we constructed three millimeter-scale sensor nodes as shown in Fig. 6. The sensors are based on a chip set of 8 die that 
contain temperature sensing, imaging, pressure reading, processor/memory, power management, harvester, near- and far-field radios, and several battery and solar cell sizes. Each sensor uses a subset of die connected in a stacked fashion and communicating via Mbus.

\section{Conclusions}

The IoT application space offers an unprecedented opportunity for the semiconductor industry. However, its diversity and severe power constraints pose serious challenges to the full adoption of IoT. In this paper, we examined the characteristics of the IoT design space and reviewed low power circuit techniques and a modular design approaches. Finally, we showed three millimeter sensor systems constructed from an 8 die chip set.

\begin{tabular}{|c|c|c|}
\hline Sensor Property & Sub categories & Range of values \\
\hline \multirow{3}{*}{$\begin{array}{l}\text { Sensing / Actuation } \\
\text { Modality }\end{array}$} & Type & $\begin{array}{l}\text { Pressure, temperature, strain, chemical, } \\
\text { imaging, sound, etc. }\end{array}$ \\
\hline & Duty Cycle & Continuous $\leftrightarrow$ Infrequent, on-demand \\
\hline & Trigger & $\begin{array}{l}\text { Timer based (accuracy?); event in monitored } \\
\text { value; external trigger } \\
\text { (wakeup radio, battery condition, etc.) }\end{array}$ \\
\hline $\begin{array}{l}\text { Computational } \\
\text { Performance } \\
\end{array}$ & & Sub-kHz $\leftrightarrow 100$ 's of $\mathrm{MHz}$ \\
\hline Form Factor / Size & & Submillimeter $\leftrightarrow$ tens of centimeter scale \\
\hline $\begin{array}{l}\text { Energy Source / } \\
\text { Life Time }\end{array}$ & & $\begin{array}{l}\text { Wired (permanently, intermittently); } \\
\text { battery (primairy, secondary); } \\
\text { energy scavenging (continuous or as available); } \\
\text { lifetime of hrs, days, years }\end{array}$ \\
\hline \multirow{3}{*}{$\begin{array}{l}\text { Communication } \\
\text { Connectivity }\end{array}$} & Type & $\begin{array}{l}\text { Wired vs. wireless (communication distance, } \\
\text { communication standard) }\end{array}$ \\
\hline & \begin{tabular}{|l|} 
Data Rate / \\
Packet Size \\
\end{tabular} & kbps $\leftrightarrow$ Gbps; Bytes $\leftrightarrow$ MBytes \\
\hline & $\begin{array}{l}\text { Communication } \\
\text { Interval }\end{array}$ & $\begin{array}{l}\text { Continuous } \leftrightarrow \text { Infrequent; triggered by } \\
\text { monitored event or external request }\end{array}$ \\
\hline
\end{tabular}

Table 1. Categorization of IoT application space in terms of 4 key properties
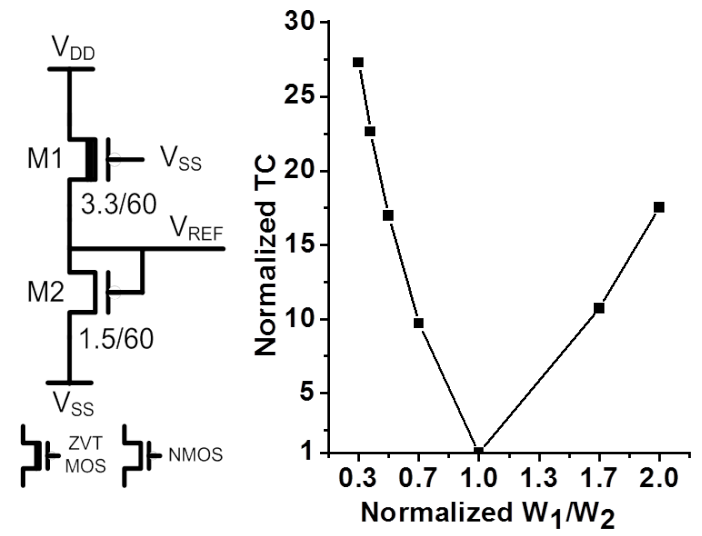

$V_{\text {ref }}=\frac{m_{1} m_{2}}{m_{1}+m_{2}}\left(V_{T H 2}-V_{T H 1}\right)+\frac{m_{1} m_{2}}{m_{1}+m_{2}} V_{T} \ln \left(\frac{\mu_{1} C_{o x 1} W_{1} L_{2}}{\mu_{2} C_{o x 2} W_{2} L_{1}}\right)$

Figure 2. Low power voltage reference with pA current draw

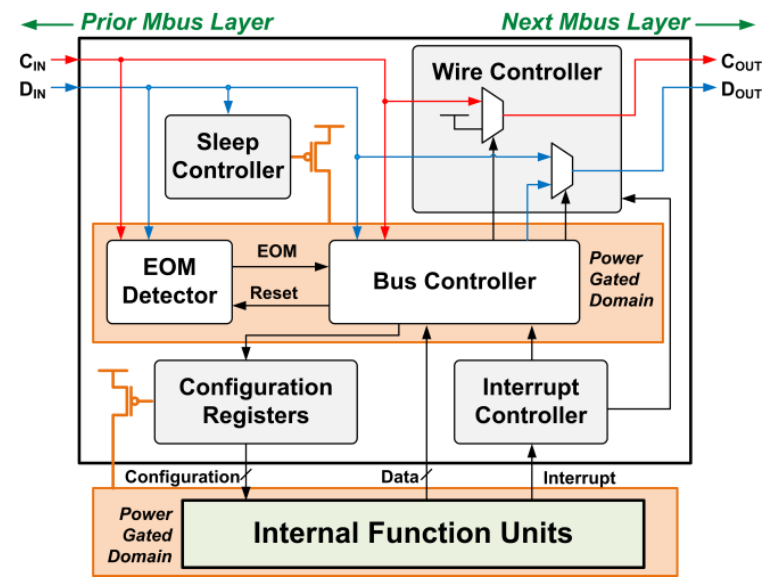

Figure 5. MBus design for low power IoT die-to-die communication

\section{Acknowledgements}

This work is funded in part by the SRC/MARCO/DARPA TerraSwarm Research Center and NSF.

\section{References}

[1] Yu, International SPE Conference, \#159583, 2012

[2] R.A.MacArthur, et al., Canadian Journal of Zoology, vol. 51. 1973

[3] M. Seok, et al., JSSC, vol. 47, pgs. 2534-2545, 2012

[4] D. Yoon, et al., ISSCC 2012 [5] W. Jung, et al., ISSCC 2014

[6] SPI Block Guide V03.06, Freescale Semiconductor

[7] http://www.nxp.com/documents/user_manual/UM10204.pdf

[8] G. Kim, et al., VLSI-Symp. 2014

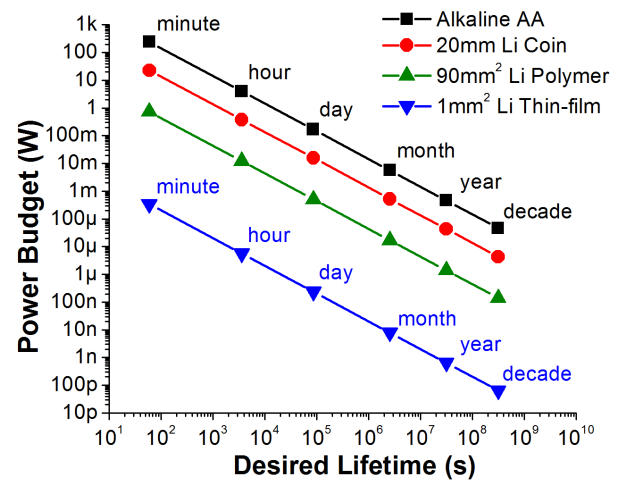

Figure 1. Average power draw constraint as a function of lifetime and battery size

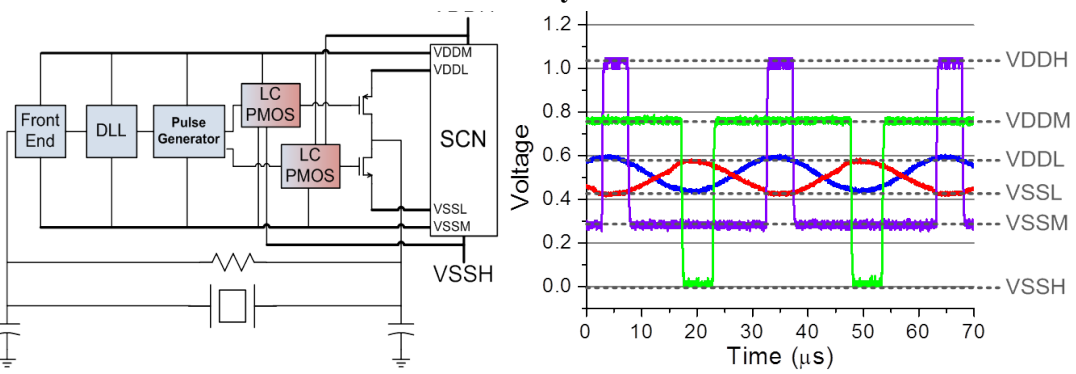

Figure 3. A 5.5nW crystal oscillator with low swing oscillation amplitude

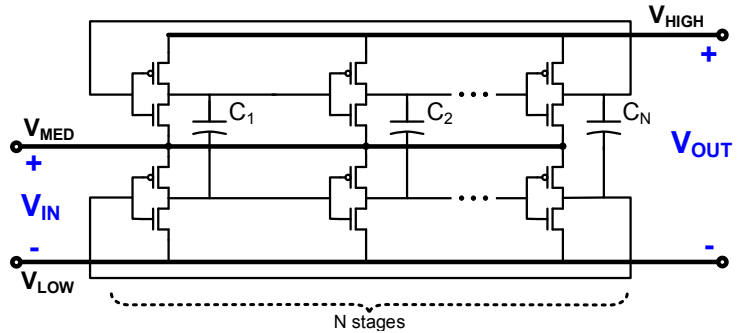

Figure 4. Voltage doubler with integrated oscillator and $>70 \%$ efficiency from $1 \mathrm{nA}-300 \mu \mathrm{A}$

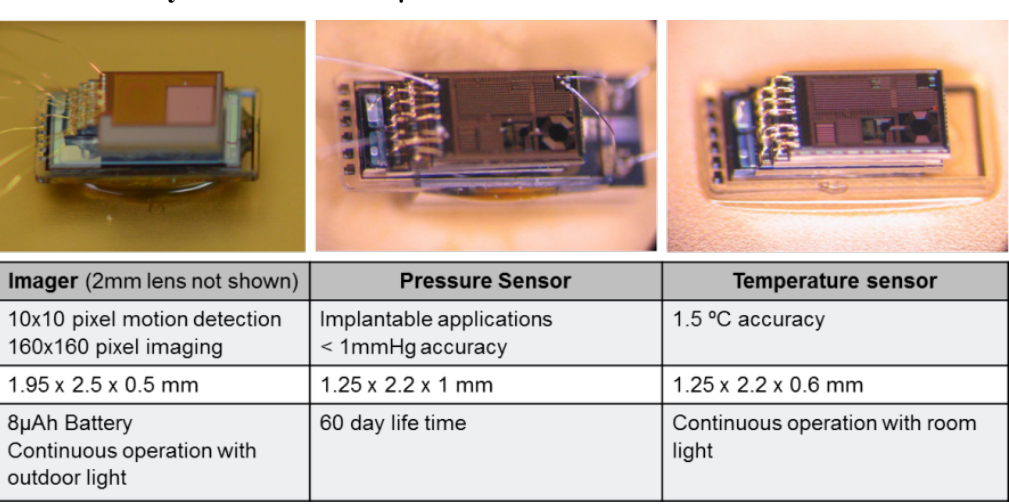

Figure 6. Millimeter sensor designs constructed using low power die connected through MBus 